\title{
TOPOLOGICAL ENTROPY FOR FINITE INVARIANT SUBSETS OF $Y$
}

\author{
SHIHAI LI AND XIANGDONG YE
}

\begin{abstract}
Let $Y$ be the space $\left\{z \in \mathbf{C}: z^{3} \in[0,1]\right\}$ with a metric defined by the arc length. Suppose that $f$ is a continuous map from $Y$ to itself and $P$ is a finite $f$-invariant subset. In this paper we construct a continuous map $C_{P}$ from $Y$ to itself satisfying $\left.C_{P}\right|_{P}=\left.f\right|_{P}$ which achieves the infimum topological entropies of continuous maps from $Y$ to itself which agree with $f$ on $P$.
\end{abstract}

\section{INTRODUCTION}

By a graph we mean a connected compact one-dimensional branched manifold. A tree is a graph without cycles. A triod is a tree with only three branches. Here we take $Y$ as the triod $\left\{z \in \mathbf{C}: z^{3} \in[0,1]\right\}$ along with a metric defined by the arc length. See [ACG], [ALM1,2], [AM], [AY], [Bald1,2], [BC], [BGMY], [B1] and [LM] for some results concerning the dynamical properties of graph maps, see $[\mathrm{FM}]$ for the relation between disk homeomorphisms and tree maps.

Topological entropy is a topological invariant which measures how a map mixes up the points of the space by iteration. For a definition and the fundamental properties of topological entropy see, for instance, [DGS].

Let $P=\left\{p_{1}<\cdots<p_{n}\right\}$ with $n \in \mathbf{N}$ be a finite subset of interval $I=[0,1]$ and $\tau$ be a map from $P$ into itself, then by [BGMY] the piecewise linear map $C_{P}$ defined by $C_{P}\left(p_{i}\right)=\tau\left(p_{i}\right)$ and $C_{P}\left(\left[0, p_{1}\right]\right)=\left\{\tau\left(p_{1}\right)\right\}, C_{P}\left(\left[p_{n}, 1\right]\right)=$ $\left\{\tau\left(p_{n}\right)\right\}$ achieves the infimum of the topological entropies of continuous maps from $I$ to itself which agree with $\tau$ on $P$. Hence in some sense we know the simplest model of maps from $I$ into itself with a common finite invariant subset of $I$.

A similar problem has been raised for more than ten years. If we replace the interval $I$ by the space $Y$, which map can achieve the minimum entropy of the continuous maps that agree with a map $\tau$ on a given finite subset of $Y$ ? It is natural for one to think of the piecewise monotone maps on the given set. But the node 0 plays a very important role in mixing up the points in $Y$, while the itineraries of 0 are untraceable. We will solve the problem by considering two cases. First, the node 0 is in the given set, and second, 0 is not. In the first case, the itineraries of 0 are determined by $P$ and $\tau$, the minimum entropy is achieved by the piecewise monotone map.

Received by the editors September 24, 1993.

1991 Mathematics Subject Classification. Primary 58F03; Secondary 58F08, 26A18.

Key words and phrases. Minimum topological entropy, triod, tree. 
In the second case we compare the entropies of maps according to the itineraries of 0 . Let $P$ be a finite subset of $Y$, and $\tau$ be a map on $P$. We denote by $\mathscr{A}$ the set of continuous maps from $Y$ to itself which agree with $\tau$ on $P$. We solve the second case as follows. First we find a collection of countably many continuous maps in $\mathscr{A}$ which either map 0 to itself or map 0 to a point in $P$ after some iterations. Then we show that the entropy of each map $f \in \mathscr{A}$ is bigger than or equal to the topological entropy of some map in the collection. Furthermore we compare the topological entropies of the maps in the collection and find the one which achieves the minimum topological entropy.

To state the main result we need some notations. Each connected component of $Y \backslash\{0\}$ is called a branch of $Y$ and is denoted by $b r_{i}, 1 \leq i \leq 3$. For $X \subset Y,[X]$ stands for the convex hull of $X$. Hence $[a, b]$ will be the convex hull of $\{a, b\}$ and $(a, b)$ will be the set $[a, b] \backslash\{a, b\}$ for every pair $a, b \in Y$. The closure of each connected component of $[P] \backslash P$ will be called a $P$-component. When $P$ lies on three branches and $0 \notin P$, the closure of the $P$-component which contains 0 will be denoted by $s m$, and furthermore we denote by $s m_{i}$ the unique point from $P$ in $s m \cap b r_{i}$ for $i=1,2,3$ and $s m^{\tau}=\left[\left\{\tau\left(s m_{1}\right), \tau\left(s m_{2}\right), \tau\left(s m_{3}\right)\right\}\right]$. Clearly $s m=\left[\left\{s m_{1}, s m_{2}, s m_{3}\right]\right\}$.

Let $f \in C(Y, Y)$ and $Q$ be a finite subset of $Y$. We say that $f$ is piecewise linear with respect to $Q$ if $f$ maps each connected component of $Y \backslash[Q]$ to one point and $f$ is linear on the closure of each connected component of $[Q] \backslash(Q \cup\{0\})$ when $Q$ lies on three branches or $0 \in Q, f$ is linear on the closure of each connected component of $[Q] \backslash Q$ in the rest cases. We define a piecewise linear map $C_{P}$ with respect to $P$ as follows.

(1) If $0 \in P$ or $P$ lies on at most two branches we shall denote by $C_{P} \in \mathscr{A}$ the unique piecewise linear map with respect to $P$ from $Y$ into itself.

(2) If $0 \notin P$ and both $s m$ and $s m^{\tau}$ are homeomorphic to $Y$ we shall denote by $C_{P} \in \mathscr{A}$ the piecewise linear map with respect to $P$ such that $C_{P}(0)=0$.

(3) If $0 \notin P, s m$ is homeomorphic to $Y$ and $s m^{\tau}$ is not homeomorphic to $Y$ we shall denote by $C_{P} \in \mathscr{A}$ the piecewise linear map with respect to $P$ such that $C_{P}(0)=\tau\left(s m_{i_{0}}\right)$, where $i_{0} \in\{1,2,3\}$ satisfying $\tau\left(s m_{i_{0}}\right) \in$ $\left[\tau\left(s m_{i_{1}}\right), \tau\left(s m_{i_{2}}\right)\right]$ and $i_{1} \neq i_{2} \in\{1,2,3\} \backslash\left\{i_{0}\right\}$.

With these notations we can now state our main results.

Main Theorem. Let $P$ be a finite subset of $Y$ and $\tau$ be an invariant map defined on $P$. Then $C_{P}$ achieves the minimum topological entropy of continuous maps from $Y$ to $Y$ which agree with $\tau$ on $P$.

The second author would like to thank Professor L1. Alsedà for mentioning the problem and Professor S. Baldwin for talking about his preliminary reports [Bald2] by which some method used in the paper was inspired. He also thanks Professor A. Verjovsky for helpful comments.

\section{Preliminaries}

The topological entropy for general graph maps was discussed in [LM]. The following lemma is one of the main result of it. To state the lemma we need a notion. Let $X$ be a topological space and $f: X \rightarrow[0, \infty]$. We say that $f$ is lower-semicontinuous if for each $x \in X$ and $a<f(x)$ there is a neighborhood $U$ of $x$ such that $f(y)>a$ for every $y \in U$. 
Lemma 2.1. Topological entropy, as a function of a continuous map of a graph into itself, is lower semicontinuous.

The following lemma concerning the topological entropy of tree maps is an analogy to the interval case [BGMY]. Its proof can be derived from [BC] or [ALM2]. We shall use $h(\cdot)$ to denote the topological entropy.

Lemma 2.2. Let $T$ be a tree and $f \in C(T, T)$. Assume that $P$ is a finite $f$-invariant subset of $X$ containing all the vertices and $f$ satisfies that it maps each connected component of $T \backslash[P]$ to one point. Let $I_{k}, 1 \leq k \leq m$, be the closure of connected components of $T \backslash P$. Define a 0-1 matrix $M=\left(m_{i j}\right)_{m \times m}$ in the following way.

Then

$$
m_{i j}=1 \text { if and only if } f\left(I_{i}\right) \supset I_{j} .
$$

$$
h(f) \geq \max \{0, \log \lambda\},
$$

where $\lambda$ is the biggest eigenvalue of $M$. The equality holds if $f$ is monotonic on each $I_{j}$, i.e., $f^{-1}(x)$ is connected for each interior point of $I_{j}$.

The next lemma is one of the basic properties of topological entropy. Its proof can be found, for instance, in [DGS].

Lemma 2.3. (1) Let $X$ be a compact metric space and $f \in C(X, X)$. Then $h(f)=h\left(\left.f\right|_{f(X)}\right)$.

(2) Let $Z_{i}$ be compact metric spaces and $f_{i} \in C\left(Z_{i}, Z_{i}\right)$ for $i=1,2$. If $\left(Z_{1}, f_{1}\right)$ is an extension of $\left(Z_{2}, f_{2}\right)$ or $\left(Z_{2}, f_{2}\right)$ is a factor of $\left(Z_{1}, f_{1}\right)$, i.e., there exists $f \in C\left(Z_{1}, Z_{2}\right)$ such that $f_{2} \circ g=g \circ f_{1}$, then $h\left(f_{1}\right) \geq h\left(f_{2}\right)$. Furthermore if $f$ is a homeomorphism then $h\left(f_{1}\right)=h\left(f_{2}\right)$.

(3) If $S_{1}, S_{2}$ are two closed $f$-invariant subsets of $f$, then

$$
h(f) \geq \max \left\{h\left(\left.f\right|_{S_{1}}\right), h\left(\left.f\right|_{S_{2}}\right)\right\} .
$$

Furthermore if $S_{1} \cap S_{2}=\varnothing$ and $S_{1} \cup S_{2}=X$ then $h(f)=\max \left\{h\left(f \mid S_{1}\right)\right.$, $\left.h\left(\left.f\right|_{S_{2}}\right)\right\}$.

To prove the Main Theorem we shall identify the points in $Y$ so that the induced map on the quotient space has some nice properties. We will prove a lemma which shows that under our identification the quotient space is not a complicated space. To state the lemma we start with some notations.

By a continuum we mean a connected compact metric space. A continuum is called $a$ dendrite if it is uniquely arcwise connected and locally connected. $A$ decomposition $\mathbf{D}$ of a space $X$ is a collection of non-empty closed pairwise disjoint subsets of $X$ such that their union is $X$. We may define an equivalence relation on $X$ by the decomposition in the following way: $x \sim y$ if and only if $x, y \in B$ for some $B \in \mathbf{D}$. We say a decomposition is upper semicontinuous if the projection $p: X \rightarrow X / \sim$ is a closed map. For an equivalence relation $\sim$ on $X$ we will use $\tilde{x}$ to denote the equivalence class of $x$. Let $f: X \rightarrow X$ be a continuous map. For each pair $B_{1}, B_{2}$ of subsets of $X$ we say $B_{1} f$-covers $B_{2}$ if $f\left(B_{1}\right) \supset B_{2}$. Furthermore we will use $[x, y]$ to denote the unique arc connecting $x$ and $y$ for any pair $x, y$ in a dendrite.

Let $X$ be a dendrite and $f \in C(X, X)$, and $S_{1}, S_{2}$ be two closed connected subsets of $X$. For each $k \in \mathbf{N}$ we define an equivalence relation on $X$ in the following way: $x \sim y$ if and only if $x, y \in S_{1} \cap f^{-k}(z)$ for some $z \in S_{2}$, then we have 
Lemma 2.4. If $S_{1} \cap f^{-k}\left(S_{2}\right)$ is connected, then the quotient space $X / \sim$ is a dendrite. Furthermore if $X$ is a tree then $X / \sim$ is also a tree.

Proof. Let $p: X \rightarrow X / \sim$ be the projection. We claim that $p$ is closed. In fact let $B$ be a closed subset of $X$ we need to show that $p^{-1} p(B)$ is a closed subset of $X$. This follows from the observation that if $x_{i} \sim y_{i}$ with $y_{i} \in B$ then any limit points of $\left\{x_{i}\right\}$ is equivalent to some point of $B$. As $p$ is a closed map then the decomposition given by the equivalence relation is upper semicontinuous. By Theorem 1 [K, pp. 64-65] $X / \sim$ is a compact metric space. As $p$ is continuous, $X / \sim$ is locally connected [K, pp. 256-257]. Hence if we can show that $X / \sim$ is uniquely arcwise connected then $X / \sim$ is a dendrite.

To show that $X / \sim$ is uniquely arcwise connected we only need to prove that $p\left(S_{1} \cap f^{-k}\left(S_{2}\right)\right)$ is uniquely arcwise connected. Define $f^{*}: p\left(S_{1} \cap f^{-k}\left(S_{2}\right)\right) \rightarrow$ $f^{k}\left(S_{1} \cap f^{-k}\left(S_{2}\right)\right)$ in the following way: $f^{*}([x])=f^{k}(x)$ for each $x \in S_{1} \cap$ $f^{-k}\left(S_{2}\right)$. It is checked that $f^{*}$ is a bijection. As $\left.f^{*} \circ p\right|_{S_{1} \cap f^{-k}\left(S_{2}\right)}=\left.f^{k}\right|_{S_{1} \cap f^{-k}\left(S_{2}\right)}$ we get that $f^{*}$ is continuous. Since $p$ is continuous and $\left.f^{k}\right|_{S_{1} \cap f^{-k}\left(S_{2}\right)}$ is a closed map it implies that $f^{*}$ is a closed map. Hence $f^{*}$ is a homeomorphism from $p\left(S_{1} \cap f^{-k}\left(S_{2}\right)\right)$ onto $f^{k}\left(S_{1} \cap f^{-k}\left(S_{2}\right)\right) \subset X$. Hence $p\left(S_{1} \cap f^{-k}\left(S_{2}\right)\right)$ is uniquely arcwise connected.

The last statement of the lemma follows from the above proof easily.

Notice that if $S_{2}=f^{k}\left(S_{1}\right)$ then $S_{1} \subset f^{-k}\left(S_{2}\right)$. Since $S_{1}$ is connected, the condition of Lemma 2.4 is satisfied. Thus $X / \sim$ is a dendrite.

The last lemma which we want to prove in this section is the following. As usual we need some notations. Let $X$ be a compact metric space and $f \in$ $C(X, X)$. For a point or subset of $X, A$, the orbit of $A$ under $f$ is the set $O(A, f)=\{A, f(A), \ldots$,$\} . The \omega$-limit set of $x \in X$ is the set $\{y \in X$ : there are $n_{i} \in N$ such that $\left.f^{n_{i}}(x) \rightarrow y\right\}$. A point $x \in X$ is said to be $a$ nonwandering point of $f$ if for any neighborhood $V_{x}$ of $x$ there exists $n \in N$ such that $f^{n}\left(V_{x}\right) \cap V_{x} \neq \varnothing$. The set of nonwandering points of $f$ will be denoted by $\Omega(f)$. A well-known fact about $\Omega(f)$ is that $h(f)=h\left(\left.f\right|_{\Omega(f)}\right)$. By a retraction from a dendrite $T$ onto its closed connected subspace $T_{1}$ we mean a continuous map $r$ such that $r(x)=x$ for each $x \in T_{1}$ and $r$ maps each connected component of $T \backslash T_{1}$ to one point.

Lemma 2.5. Let $T$ be a dendrite and $T_{1}$ be a subdendrite such that $T \backslash T_{1}$ only has finitely many connected components. Let $f \in C(T, T)$ and $r$ be a retraction from $T$ onto $T_{1}$ then $h(f) \geq h\left(\left.r \circ f\right|_{T_{1}}\right)$.

Proof. We only need to show this if $T \backslash T_{1}$ has only one connected component $C$. Let $\{a\}=\bar{C} \cap T_{1}$. Denote $g=\left.r \circ f\right|_{T_{1}}$. We claim that (1) if $O(a, f) \subset$ $T_{1}$ then $\Omega(g)$ is also an $f$-invariant set, (2) if $O(a, f) \not \subset T_{1}$ then $B=$ $\Omega(g) \backslash O(a, g)$ is an $f$-invariant set and $O(a, g)$ is an isolated subset of $\Omega(g)$.

To prove the claim firstly we assume that $O(a, f) \subset T_{1}$. Let $x \in \Omega(g)$. If $f(x) \notin T_{1}$ then there is a neighbourhood $V_{x}$ of $x$ in $T$ such that $V_{x} \subset T_{1}$, and $f\left(V_{x}\right) \subset C$. Then $g\left(V_{x}\right)=a$. As $x \in \Omega(g)$ we have that $x \in \omega(a, g)$. Hence $f(x) \in \omega(a, g) \subset T_{1}$, a contradiction. Hence $f(x) \in T_{1}$ for all $x \in \Omega(g)$. That is to say that $f(x)=g(x) \in \Omega(g)$ for all $x \in \Omega(g)$. Hence

$$
h(f) \geq h\left(\left.f\right|_{\Omega(g)}\right)=h\left(\left.g\right|_{\Omega(g)}\right)=h(g) .
$$

Now suppose that $O(a, f) \not \subset T_{1}$. Then there is $i \in \mathbf{N}$ such that $a, f(a), \ldots$, $f^{i-1}(a) \in T_{1}$ and $f^{i}(a) \in C$. Hence $a$ is a periodic orbit of $g$. Assume that 
there is $x \in B$ such that $f(x) \in C$. Since $x \in \Omega(g)$, an arbitrary neighborhood $U$ of $x$ has an image intersecting $U$. However, if $U$ is small enough, then it is mapped entirely to $a$, so the orbit of $a$ has to intersect $U$. Since the orbit of $a$ is finite and $U$ can be arbitrarily small, it follows that $x \in O(a, g)$, a contradiction. Hence $f(x) \in T_{1}$ for all $x \in B$. To show that $B$ is an $f$-invariant subset it remains to show that $f(x) \notin O(a, g)$, for each $x \in B$. Assume the contrary, that is there is $x \in B$ such that $f(x) \in O(a, g)$. This implies that there is $j \leq i$ such that $x, f(x), \ldots, f^{j-1}(x) \in T_{1}$ and $f^{j}(x) \in C$. By the same reason as before we have $x \in \omega(a, g)$, a contradiction.

At last we show that $O(a, g)$ is an isolated set of $\Omega(g)$. If this is not the case then there are points $x_{n} \in B, x \in O(a, g)$ such that $x_{n} \rightarrow x$. Then there is $x_{n_{0}}$ such that $f\left(x_{n_{0}}\right) \in C$. This is not true by the fact that $B$ is an $f$-invariant set.

As $B$ and $O(a, g)$ are both closed $g$-invariant sets, and $\Omega(g)=B \cup O(a, g)$ we get the following inequality by using Lemma 2.3 .

$$
\begin{aligned}
h(f) & =h\left(\left.f\right|_{\Omega(f)}\right) \geq h\left(\left.f\right|_{B}\right)=h\left(\left.g\right|_{B}\right) \\
& =\max \left(h\left(\left.g\right|_{B}\right), h\left(\left.g\right|_{O(a, g)}\right)=h\left(\left.g\right|_{\Omega(g)}\right)=h(g) .\right.
\end{aligned}
$$

Hence we are done.

\section{Proof OF THE MAIN THEOREM}

With the preparation in section 2 we are now at the stage to prove the Main Theorem. Notice that if $0 \in P$ or $P$ ies on at most two branches, then by Lemma 2.5 and Lemma 2.2 $C_{P}$ achieves the toplogical entropy of $P$. So we only consider the case that $0 \notin P$ and $P$ lies on three branches. For $f \in \mathscr{A}$ we can further divide this case into two subcases, namely $f(0) \notin \operatorname{int}(s m)$ and $f(0) \in \operatorname{int}(s m)$ where int $(s m)$ is the interior of $s m$. When $f(0) \notin \operatorname{int}(s m)$, there are still two possibilities, i.e., $s m^{\tau}$ is or is not homeomorphic to $Y$. In order to compare the entropies of $f$ and $C_{P}$, we need compare the entropies of $f$ and the following maps in respective cases.

If $s m^{\tau}$ is homeomorphic to $Y$ denote by $f_{i 0} \in \mathscr{A}$ the piecewise linear map with respect to $P$ satisfying $f_{i 0}(0)=s m_{i}$ for $i=1,2,3$.

If $s m^{\tau}$ is not homeomorphic to $Y$, we have the following two cases which will occur when we make identification later. $C_{P^{\prime}}$ in the following is piecewise linear with respect to $P^{\prime}$ (see Section 1). Let $i_{0}, i_{1}, i_{2} \in\{1,2,3\}$ be the integers such that $\tau\left(s m_{i_{0}}\right) \in\left[\tau\left(s m_{i_{1}}\right), \tau\left(s m_{i_{2}}\right)\right]$.

(a) $\tau\left(s m_{i_{0}}\right) \in\left(\tau\left(s m_{i_{1}}\right), \tau\left(s m_{i_{2}}\right)\right)$.

In this case we define $P^{\prime}$ to be $\left(P \backslash\left\{s m_{i_{0}}\right\}\right) \cup\{0\}$ and

$$
C_{P^{\prime}}(x)= \begin{cases}\tau(x), & \text { if } x \in P \backslash\left\{s m_{i_{0}}\right\} \text { and } \tau(x) \neq s m_{i_{0}} \\ 0, & \text { if } x \in P \backslash\left\{s m_{i_{0}}\right\} \text { and } \tau(x)=s m_{i_{0}} \\ \tau\left(s m_{i_{0}}\right), & \text { if } x=0 \text { and } \tau\left(s m_{i_{0}}\right) \neq s m_{i_{0}} \\ 0, & \text { if } x=0 \text { and } \tau\left(s m_{i_{0}}\right)=s m_{i_{0}} .\end{cases}
$$

(b) $\tau\left(s m_{i_{0}}\right)=\tau\left(s m_{i_{1}}\right)$ or $\tau\left(s m_{i_{0}}\right)=\tau\left(s m_{i_{2}}\right)$.

By changing the labeling of $s m_{i_{j}}, j=1,2$, we may assume that $\tau\left(s m_{i_{0}}\right)=$ 
$\tau\left(s m_{i_{1}}\right)$. We define $P^{\prime}$ to be $\left(P \backslash\left\{s m_{i_{0}}, s m_{i_{1}}\right\}\right) \cup\{0\}$ and

$$
C_{P^{\prime}}(x)= \begin{cases}\tau(x), & \text { if } x \in P \backslash\left\{s m_{i_{0}}, s m_{i_{1}}\right\} \text { and } \tau(x) \neq s m_{i_{0}}, s m_{i_{1}} \\ 0, & \text { if } x \in P \backslash\left\{s m_{i_{0}}, s m_{i_{1}}\right\} \text { and } \tau(x)=s m_{i_{0}} \text { or } s m_{i_{1}} \\ \tau\left(s m_{i_{0}}\right), & \text { if } x=0 \text { and } \tau\left(s m_{i_{0}}\right) \neq s m_{i_{0}}, s m_{i_{1}} \\ 0, & \text { if } x=0 \text { and } \tau\left(s m_{i_{0}}\right)=s m_{i_{0}} \text { or } s m_{i_{1}} .\end{cases}
$$

In the following lemma we will show that $h(f) \geq h\left(f_{i 0}\right)$ for some $i \in$ $\{1,2,3\}$ when $s m^{\tau}$ is homeomorphic to $Y$ and $f(0) \notin \operatorname{int}(s m)$. In the proof we identify certain points first to create a quotient space, and an induced map on the quotient space. A subspace of the quotient space is homeomorphic to $Y$. And the homeomorphism induces a continuous map on $Y$ which maps the node 0 to a point in $P$. Then by Lemma 2.2, this map has no smaller entropy than $f_{i 0}$ has for a prefixed $i \in\{1,2,3\}$. Notice that by Lemma 2.3 the entropy of $f$ is no smaller than the entropy of the induced map on the quotient space which is further bigger than or equal to the entropy of the composition of the induced map with a retraction by Lemma 2.5. Then by Lemma 2.3 again on the equality of the entropies of the conjugated maps and by Lemma 2.2 we are done for this case.

When $s m^{\tau}$ is not homeomorphic to $Y$ and $f(0) \notin \operatorname{int}(s m)$ using the same idea as above we can prove that the entropy of $f$ is no smaller than the entropy of $C_{P^{\prime}}$. By some calculation we can see that the entropy of $C_{P^{\prime}}$ is equal to the entropy of $C_{P}$. Therefore $h(f) \geq h\left(C_{P}\right)$.

Lemma 3.1. Suppose that $f(0) \notin \operatorname{int}(s m)$. If $s m^{\tau}$ is homeomorphic to $Y$ then $h(f) \geq h\left(f_{i 0}\right)$ for some $i \in\{1,2,3\}$. If $s^{\tau}$ is not homeomorphic to $Y$ then $h(f) \geq h\left(C_{P}\right)$.

Proof. Case 1: $s^{\tau}$ is homeomorphic to $Y$.

Suppose that $f(0) \in[a, b]$ with $a, b \in P$ and $(a, b) \cap P=\varnothing$. Without loss of generality we suppose that $f(0) \in b r_{1}$. Let $i_{1}, i_{2}$ be integers such that $\tau\left(s m_{i_{1}}\right)$ and $\tau\left(s m_{i_{2}}\right)$ are not in $b r_{1}$. As $\left[0, s m_{i_{1}}\right]$ and $\left[0, s m_{i_{2}}\right]$ both $f$-cover $\left[s m_{1}, 0\right]$ there exist $x_{j} \in\left(0, s m_{i_{j}}\right)$ with $j=1,2$ such that

$$
f\left(x_{1}\right)=f\left(x_{2}\right)=s m_{1} \text { and } s m_{1} \notin f\left(0, x_{1}\right) \cup f\left(0, x_{2}\right) .
$$

Define an equivalence relation on $Y$ in the following way: $x \sim y$ if and only if $x, y \in\left[x_{1}, x_{2}\right]$ and $f(x)=f(y)$. Then we get a quotient space $Y / \sim$, a projection $p: Y \rightarrow Y / \sim$ and a continuous map $\tilde{f}$ from $Y / \sim$ to itself with $p \circ f=\tilde{f} \circ p$. Then $\tilde{f}\left(\tilde{x}_{1}\right)=s m_{1}$. By Lemma 2.3, $h(f) \geq h(\tilde{f})$.

By the remark after Lemma $2.4, Y / \sim$ is a tree. $\left[\tilde{x}_{1}, \tilde{0}\right]$ is thus homeomorphic to an interval. Notice that $p\left(\left[x_{1}, x_{2}\right]\right)$ is homeomorphic to $f\left(\left[x_{1}, x_{2}\right]\right)$ which is an interval by the choice of $x_{1}$ and $x_{2} . p\left(\left[x_{1}, x_{2}\right]\right)$ is an arc that contains $\left[\tilde{x}_{1}, \tilde{0}\right] . p\left(\left[x_{1}, x_{2}\right]\right) \backslash\left[\tilde{x}_{1}, \tilde{0}\right]$ is a branch joining the rest of $Y / \sim$ at 0.

Let $r: Y / \sim \rightarrow(Y / \sim) \backslash\left(p\left(\left[x_{1}, x_{2}\right]\right) \backslash\left[\tilde{x}_{1}, \tilde{0}\right]\right)$ be a retraction. Then by Lemma 2.5 we have that

$$
h(f) \geq h(\tilde{f}) \geq h\left(\left.r \circ \tilde{f}\right|_{r(Y / \sim)}\right)
$$

Moreover one can see that $r(Y / \sim)$ is homeomorphic to $Y$ by the definition of $r .\left.\quad r \circ \tilde{f}\right|_{r(Y / \sim)}$ is conjugate to a map from $\mathscr{A}$ which maps 0 to $s m_{1}$. 
Therefore we have $h(f) \geq h\left(\left.r \circ \tilde{f}\right|_{r(Y / \sim)}\right) \geq h\left(f_{10}\right)$ by Lemma 2.2 and Lemma 2.3.

For the same reason if $f(0) \in b r_{i}$ then $h(f) \geq h\left(f_{i 0}\right), i=2,3$.

Case 2: $s m^{\tau}$ is not homeomorphic to $Y$.

Subcase 1. $\tau\left(s m_{i_{0}}\right) \in\left(\tau\left(s m_{i_{1}}\right), \tau\left(s m_{i_{2}}\right)\right)$. Let $R_{1}, R_{2}$ be the closures of two connected components of $Y \backslash \tau\left(s m_{i_{0}}\right)$ such that $\tau\left(s m_{i_{j}}\right) \in R_{j}$ for $j=1,2$.

If $f(0) \in R_{1}$ then $\left[0, s m_{i_{0}}\right]$ and $\left[0, s m_{i_{2}}\right]$ both $f$-cover $\left[f(0), \tau\left(s m_{i_{0}}\right)\right]$. Hence there exist $x_{1} \in\left[0, s m_{i_{0}}\right]$ and $x_{2} \in\left[0, s m_{i_{2}}\right]$ such that $f\left(x_{1}\right)=f\left(x_{2}\right)=$ $\tau\left(s m_{i_{0}}\right)$. Then we define an equivalence relation on $Y$ as the same as in the Case 1 and proceed with the same argument. Finally we get $h(f) \geq h\left(C_{P^{\prime}}\right)$.

In case $f(0) \in R_{2}$ the lemma can be proved similarly.

Subcase 2. $\tau\left(s m_{i_{0}}\right)=\tau\left(s m_{i_{1}}\right)$ or $\tau\left(s m_{i_{2}}\right)$. In this case we set $S_{1}=\left[s m_{i_{0}}, s m_{i_{1}}\right]$ if $\tau\left(s m_{i_{0}}\right)=\tau\left(s m_{i_{1}}\right)$ and $S_{1}=\left[s m_{i_{0}}, s m_{i_{2}}\right]$ if $\tau\left(s m_{i_{0}}\right)=\tau\left(s m_{i_{2}}\right)$. Let $S_{2}=$ $f\left(S_{1}\right)$. Using the identification defined before Lemma 2.4 and the same argument as above we get that $h(f) \geq h\left(C_{P^{\prime}}\right)$.

Let $M, M^{\prime}$ be the induced matrices by $C_{P}$ and $C_{P^{\prime}}$ respectively. If $\lambda_{1}, \ldots$, $\lambda_{m}$ are eigenvalues of $M^{\prime}$ then $0, \ldots, 0, \lambda_{1}, \ldots, \lambda_{m}$ are eigenvalues of $M$. Hence $h\left(C_{P}\right)=h\left(C_{P^{\prime}}\right)$. Thus we have $h(f) \geq h\left(C_{P}\right)$.

Now we consider the situation that $f(0) \in \operatorname{int}(\mathrm{sm})$. We start with some notations.

Let $f \in \mathscr{A}$. We define $\phi$ to be a map from $\left\{s m_{1}, s m_{2}, s m_{3}\right\}$ to itself satisfying $\phi\left(s m_{i}\right)=s m_{j}$ if $\tau\left(s m_{i}\right) \in b r_{j}$. Suppose that $f(0) \in b r_{i_{0}}$. As $s m_{i_{0}}$ is an eventually periodic point of $\phi$, then there exist $p \in\{0,1,2\}$ and a periodic orbit $Q$ of $\phi$ such that either $\phi^{p}\left(s m_{i_{0}}\right) \in Q$ for $p=0$ or $\phi^{p}\left(s m_{i_{0}}\right) \in$ $Q$ but $\phi^{i}\left(s m_{i_{0}}\right) \notin Q$ for $0 \leq i \leq p-1$ for $p>0$. Suppose that $Q=$ $\left\{s m_{p+1}, \ldots, s m_{p+r}\right\}$ and $\phi\left(s m_{p+i}\right)=s m_{p+i+1(\bmod r)}$ for $0 \leq i \leq r-1$ where $r$ is the period of the points in $Q$.

Let $d$ be a metric on $Y$ and $\|\cdot, \cdot\|$ be a norm in $C(Y, Y)$. We say that $f$ maps 0 according to $\phi$ up to $k$ th iteration if

Condition 1. $f^{i}(0) \in \operatorname{int}(s m)$ for $i=0,1,2, \ldots, k, f^{k+1}(0) \in Y \backslash \operatorname{int}(s m)$. $b r_{j}$.

Condition 2. $f^{i+1}(0) \in b r_{j}$ for any pair $i, j=0,1,2, \ldots, k$ if $\phi^{i}\left(s m_{i_{0}}\right) \in$

Condition 3. $d\left(f^{p+i}(0), 0\right)<d\left(f^{p+i+r}(0), 0\right)$ for every $i$ satisfying that $0 \leq i \leq k+1-p-r$.

We will denote by $f_{00}$ the piecewise linear map from $\mathscr{A}$ satisfying that 0 is a fixed point; by $f_{i k}$ the piecewise linear map from $\mathscr{A}$ such that $f_{i k}(0) \in b r_{i}$, it maps 0 according to $\phi$ up to $k$ th iteration and $f_{i k}^{k+1}(0)=s m_{j}$ if $f_{i k}^{k+1}(0) \in b r_{j}$ for $i=1,2,3$ and $k \in N$. Such a map is uniquely determined.

We have defined $f_{i 0}$ in the case where $s m^{\tau}$ is homeomorphic to $Y$. Here we extend the definition to the case where $s m^{\tau}$ is not homeomorphic to $Y$.

Lemma 3.2. $h\left(f_{i k}\right) \geq h\left(f_{00}\right) \geq h\left(C_{P}\right)$ for $i \in\{1,2,3\}$ and $k \in \mathbf{N} \cup\{0\}$.

Proof. When $s m^{\tau}$ is homeomorphic to $Y$, by the definition of $C_{P}$ and $f_{00}$, we can see that $f_{00}=C_{P}$. Hence $h\left(f_{00}\right) \geq h\left(C_{P}\right)$. We need only to show next $h\left(f_{i k}\right) \geq h\left(f_{00}\right)$ for $i=1,2,3$ and $k \in \mathbf{N} \cup\{0\}$. By the definition of $\phi$ there are only two possibilities.

(i) $\phi$ has a periodic orbit of period 3 .

Without loss of generality we suppose that $i=1$ and $f\left(s m_{j}\right) \in b r_{j+1(\bmod 3)}$ 
for $j=1,2,3$. Let $Z$ be the connected component of $P \cup\left\{f_{i k}(0), \ldots, f_{i k}^{k}(0)\right\}$ such that $0 \in Z$ and $f(0) \in b r_{1}$. Let $z_{1}, z_{2}, z_{3}$ be the boundary points of $Z$ with $z_{j} \in b r_{j}$ for $j=1,2,3$. Then there exist $x \in\left(0, z_{1}\right)$ and $y \in\left(0, z_{2}\right)$ such that $f_{i k}(x)=f_{i k}(y)=0$. Define an equivalence relation in $Y$ as follows: $x_{1} \sim x_{2}$ if and only if $f_{i k}\left(x_{1}\right)=f_{i k}\left(x_{2}\right)$, and $x_{1}, x_{2} \in[x, y]$. Thus if we use the same argument as in Lemma 3.1 we get that $h\left(f_{i k}\right) \geq h\left(f_{(i+1(\bmod 3))(k+1)}\right)$. Hence $h\left(f_{i k}\right) \geq h\left(f_{i(k+3)}\right)$.

By Lemma 2.2 the topological entropy of $f_{i k}$ does not depend on the lengths of each connected component of $[P] \backslash(P \cup\{0\})$ so we can change the lengths such that for $0 \leq i, j \leq 3, n_{k}=3 k+j, \lim _{k \rightarrow \infty}\left\|f_{i n_{k}}, f_{00}\right\|=0$.

As topological entropy is lower semicontinuous we have $h\left(f_{i n_{k}}\right) \geq h\left(f_{00}\right)$ for $0 \leq i, j \leq 3, k \in \mathbf{N} \cup\{\mathbf{0}\}$. This completes the proof of (i). 2.

(ii) $\phi$ has three fixed points or one fixed point and a periodic orbit of period

In this case we have that $h\left(f_{i k}\right) \geq h\left(f_{i(k+1)}\right)$ or $h\left(f_{i k}\right) \geq h\left(f_{i(k+2)}\right)$. Hence by a similar argument as in (i) we have $h\left(f_{i k}\right) \geq h\left(f_{00}\right)$.

For a proof of the second case, we can use the same argument as in the proof of (i) to show $h\left(f_{i k}\right) \geq h\left(f_{00}\right)$. Hence we only need to show that $h\left(f_{00}\right) \geq$ $h\left(C_{P}\right)$.

By labeling the branches we may assume that $\tau\left(s m_{1}\right) \in\left[\tau\left(s m_{2}\right), \tau\left(s m_{3}\right)\right]$, and $\tau\left(s m_{1}\right)$ and $\tau\left(s m_{2}\right)$ lie on the same branch. Hence there exists $a \in$ $\left[0, s m_{2}\right]$ such that $f(a)=\tau\left(s m_{1}\right)$.

Define an equivalence relation on $Y$ as follows: $x \sim y$ if and only if $x, y \in$ $\left[s m_{1}, a\right] \cap f_{00}^{-1}(z)$ for some $z \in\left[\tau\left(s m_{1}\right), 0\right]$ then we have

$$
h\left(f_{00}\right) \geq h\left(C_{P^{\prime}}\right)=h\left(C_{P}\right) .
$$

Lemma 3.3. Let $f \in \mathscr{A}$ and $f(0) \in \operatorname{int}(s m)$. If $f$ maps 0 according to $\phi$ up to $k$ th iteration then $h(f) \geq h\left(C_{P}\right)$.

Proof. If $f$ maps 0 according to $\phi$ up to $k$ th iteration then there is $x \in$ $(0, f(0))$ such that $f^{k}(x)=s m_{k+1(\bmod p+r)}$ and satisfies Condition 2 and Condition 3 if we replace $f(0)$ by $x$. Note that $\widetilde{P}=P \cup\left\{x, f(x), \ldots, f^{k-1}(x)\right\}$ is an $f$-invariant set. Let $\tilde{\tau}=\left.f\right|_{\widetilde{P}}$. Then $\tilde{\tau}$ is invariant on $\widetilde{P}$. Let $\widetilde{s m}$ be the component of $[\widetilde{P}] \backslash \widetilde{P}$ containing 0 . Then $f(0) \notin \operatorname{int}(\widetilde{s m})$ by the choice of $x$. If $\widetilde{s m}^{\tilde{\tau}}$ is homeomorphic to $Y$, by Lemma 3.1 , we have $h(f) \geq h\left(\tilde{f}_{i 0}\right)$ where $\tilde{f}_{i 0}(0) \in \widetilde{s m} \backslash \operatorname{int}(\widetilde{s m})$. By Lemma 3.2, $h\left(\tilde{f}_{i 0}\right) \geq h\left(C_{\widetilde{P}}\right)$ where $\widetilde{P}$ is piecewise linear with respect to $\widetilde{P}$ and $C_{\widetilde{p}}(0)=0$. By Lemma 2.2 and Lemma 13 (p. 198) of [BC], we have $h\left(C_{\widetilde{P}}\right) \geq h\left(C_{P}\right)$. We are done in this case.

If $s m^{\tau}$ is not homeomorphic to $Y$, by Lemma 3.1 again, $h(f) \geq h\left(C_{\widetilde{P}}\right)$, where $C_{\widetilde{P}}$ is piecewise linear with respect to $\widetilde{P}$ and $C_{\widetilde{P}}(0)=\tilde{\tau}\left(\widetilde{s m}_{i_{0}}\right)$ with $\tilde{\tau}\left(\widetilde{s m}_{i_{0}}\right) \in\left[\tilde{\tau}\left(\widetilde{s m}_{i_{1}}\right), \tilde{\tau}\left(\widetilde{s m}_{i_{2}}\right)\right]$ for $i_{1} \neq i_{2} \in\{1,2,3\} \backslash\left\{i_{0}\right\}$. In this case there are three possibilities, namely $\phi$ has a periodic orbit of period 2, the other situations are similar.

Now we suppose that $\phi$ has a periodic orbit of period 2. If $x$ and $f^{2}(x)$ are on the same branch $b r_{i}$, but the $s m_{l}$ on the third branch is not mapped to $b r_{i}$, then $C_{\widetilde{P}}=f(x)$. Hence $C_{\widetilde{P}}=\tilde{f}_{j 0}$, where $b r_{j}$ contains $f(x)$. By Lemma 
2.2, Lemma 13 (p. 198) of [BC] and Lemma 3.2,

$$
h\left(C_{\widetilde{P}}\right)=h\left(\tilde{f}_{j 0}\right) \geq h\left(\tilde{f}_{00}\right) \geq h\left(f_{00}\right) \geq h\left(C_{P}\right) .
$$

If $x$ and $f^{2}(x)$ are on the same branch $b r_{i}$, and the $s m_{l}$ on the third branch is mapped to $b r_{i}$ too, then $C_{\widetilde{P}}=f^{2}(x)$. Let $\widetilde{\widetilde{P}}=\widetilde{P} \backslash\{x\}$. Then $C_{\widetilde{P}}=\tilde{\tilde{f}}_{i 0}$, where $\tilde{\tilde{f}}_{i 0}$ is defined with respect to $\tilde{\widetilde{P}}$. By Lemma 2.2 , Lemma 13 (p. 198) of $[\mathrm{BC}]$ and Lemma 3.2 again

$$
h\left(C_{\widetilde{P}}\right)=h\left(\tilde{\tilde{f}}_{i 0}\right) \geq h\left(\tilde{\tilde{f}}_{00}\right) \geq h\left(f_{00}\right) \geq h\left(C_{P}\right) .
$$

Therefore $h(f) \geq h\left(C_{\widetilde{P}}\right) \geq h\left(C_{P}\right)$.

We denote by $P(f)$ the set of periodic points of $f$.

Lemma 3.4. Let $f \in \mathscr{A}$ and $f(0) \in \operatorname{int}(s m)$. Then $h(f) \geq h\left(C_{P}\right)$.

Proof. If $f$ maps 0 according to $\phi$ up to $k$-the iteration, then by Lemma 3.3, $h(f) \geq h\left(C_{P}\right)$. If $f$ does not map 0 according to $\phi$ up to any iteration, we have the following four cases.

(i) Condition 1 holds but Condition 2 fails;

(ii) Condition 1 holds but Condition 3 fails;

(iii) Condition 1 fails but both Condition 2 and Condition 3 hold;

(iv) Condition 1 fails and at least one of Condition 2 and Condition 3 fails.

If Condition 1 holds, there is an integer $k>0$ such that $f^{i}(0) \in \operatorname{int}(s m)$ for $0 \leq i \leq k$ but $f^{k+1}(0) \in Y \backslash \operatorname{int}(s m)$. In case (i) since Condition 2 fails to hold, there must be an integer $l$ with $0 \leq l \leq k$ such that $f^{l+1}(0)$ and $\phi^{l+1}\left(s m_{i_{0}}\right)$ belong to different branches. $\left[f^{l+1}(0), \phi^{l+1}\left(s m_{i_{0}}\right)\right]$ must contain the node 0 . Thus there is a point $a \in\left[f^{l}(0), \phi^{l}\left(s m_{i_{0}}\right)\right]$ such that $f(a)=0$. Since $l \leq k$, we have $a \in \operatorname{int}(s m)$. Let $A=\left\{a, 0, f(0), \ldots, f^{l}(0)\right\}$. Then for any point $x \in A$, one of $x, f(x)$ and $f^{2}(x)$ belongs to int $([A])$. Define $B=\left\{x \in Y \backslash[A]: f^{n}(x)=x\right.$ for some integer $n>0$ and $f^{i}(x) \in Y \backslash[A]$ for $i=0,1, \ldots, n-1\}$. Then $\bar{B}$ is invariant under $f$ and $\bar{B} \cap[A]=\varnothing$ by the construction of $A$.

Let $p$ be a map from $Y$ to $Y$ such that $p([A])=\{0\}$ and $p$ is a homeomorphism from $Y \backslash[A]$ onto $Y \backslash\{0\}$. We define $\cdot f^{\prime}: Y \rightarrow Y$ by $f^{\prime}(0)=0$ and $f^{\prime}(x)=p\left(f\left(p^{-1}(x)\right)\right)$ if $x \neq 0$. Then $f^{\prime}$ is a continuous map. It is easy to check that $f^{\prime}(0)=0$ and $p(\bar{B})$ is closed and invariant under $f^{\prime}$. Moreover $\bar{P}\left(f^{\prime}\right)=p(\bar{B}) \cup\{0\}$ is the union of two closed and invariant sets.

By Lemma 2.3 and the paper [Y], $h\left(f^{\prime}\right)=h\left(\left.f^{\prime}\right|_{\bar{P}\left(f^{\prime}\right)}\right)=h\left(\left.f^{\prime}\right|_{p(\bar{B})}\right)$. Since $p$ is a homeomorphism on $\bar{B}$, we have $h\left(\left.f^{\prime}\right|_{p(\bar{B})}\right)=h\left(\left.f\right|_{\bar{B}}\right)$. And $h\left(\left.f\right|_{\bar{B}}\right) \leq h(f)$ since $\bar{B}$ is a closed and $f$-invariant subset of $Y$. Since $l \leq k$ and $a \in \operatorname{int}(s m)$, we have $[A] \subset \operatorname{int}(s m)$. Then by Lemma $2.2, h\left(f^{\prime}\right) \geq h\left(f_{00}^{\prime}\right)$ where $f_{00}^{\prime}$ fixes 0 and is piecewise linear with respect to $p(P)$. By pulling back by $p$ to a continuous map that fixes 0 and such that $P$ is invariant, by using Lemma 2.2 again, we get $h\left(f_{00}^{\prime}\right) \geq h\left(f_{00}\right)$.

In case (ii) Condition 3 fails to hold. There are two possibilities, for $0 \leq i \leq$ $k+1-p-r$, (iia) $d\left(f^{p+i}(0), 0\right)=d\left(f^{p+i+r}(0), 0\right)$, and (iib) $d\left(f^{p+i}(0), 0\right)>$ $d\left(f^{p+i+r}(0), 0\right)$. In (iia), $f^{p+i}(0)$ is a periodic point with period $r$. Let $\widetilde{P}=$ $P \cup O(0, f)$. Then by using Lemma 2.2 and Lemma 2.5 we have that $h(f)$ is bigger than or equal to the topological entropy of the piecewise linear map $f^{\prime}$ 
with respect to $\widetilde{P}$. We denote $[O(0, f)]$ by $C$. It is easy to see that $C$ is an $f^{\prime}$-invariant set. By identifying $C$ as a point, we get a quotient space which is homeomorphic to $Y$ and an induced map $f^{\prime \prime}$ which is semiconjugate to $f^{\prime}$. Moreover $f^{\prime \prime}$ fixes the node of the quotient space. Thus $h\left(f^{\prime}\right) \geq h\left(f^{\prime \prime}\right) \geq$ $h\left(f_{00}\right)$. Therefore $h(f) \geq h\left(f_{00}\right)$.

In case (iib) we denote $A=\left\{0, f(0), \ldots, f^{p+i+r}(0)\right\}$. By using the same technic as in (i) we get $h(f) \geq h\left(f_{00}\right)$.

In case (iii) let $B=[O(0, f)]$. If $[O(B, f)] \subset$ sm we denote $C=[O(B, f)]$. Then $f(C) \subset C$. Notice that if $s m_{i} \in C$ then it is a periodic point of $f$ with period 1,2 or 3 and $O\left(s m_{i}, f\right) \subset\left\{s m_{1}, s m_{2}, s m_{3}\right\}$. By a similar argument as in (iia) one can see that $h(f) \geq h\left(f^{\prime}\right) \geq h\left(f^{\prime \prime}\right) \geq h\left(f_{00}\right) \geq h\left(C_{P}\right)$ by using Lemma 2.2 and Lemma 2.3 and Lemma 3.2.

In case $[O(B, f)] \not \subset s m$ we know that there exists $i \in \mathbf{N}$ such that $y \in$ $(0, f(0))$ with $y \in f^{-i}(x)$ for some $x=s m_{j}$ as $f([0, f(0)]) \supset[0, f(0)]$. Denote $\widetilde{P}=P \cup\left\{y, f(y), \ldots, f^{i-1}(y)\right\}$. Then $\widetilde{P}$ is an $f$-invariant set. Let $i_{0}$ be the integer such that $d\left(0, f^{i_{0}}(y)\right)=\min \left\{d\left(0, f^{l}(y)\right): f^{l}(y) \in(0, f(0))\right.$ for any possible $i-1 \geq l \geq 0\}$. By Lemma 3.1 we get that $h(f) \geq h\left(f^{\prime}\right)$, where $f^{\prime}$ is defined similar to $f_{i 0}$ and $C_{P^{\prime}}$ based on the $f$-invariant set $\widetilde{P}$ and $f^{\prime}(0)=f^{j_{0}}(y)$ for some $1 \leq j_{0} \leq i$ or $\tau\left(s m_{i_{0}}\right)$, where we use the same notation $i_{0}$ as in Lemma 3.1.

It is obvious that Condition 1 for $f^{\prime}$ must hold. Then by Case (i) or Case (ii) or Lemma 3.3 we know that $h(f) \geq h\left(f^{\prime}\right) \geq h\left(C_{P}\right)$.

In case (iv), we can choose $A$ as in either (i) or (ii). Since $f^{i}(0) \in \operatorname{int}(s m)$ for all $0 \leq i,[A]$ is still contained in $\operatorname{int}(s m)$. By similar arguments, we have $h(f) \geq h\left(C_{P}\right)$.

To sum up we have proved that $h(f) \geq h\left(C_{P}\right)$.

Proof of the Main Theorem. By Lemmas 3.1-3.4 it is easy to see that the main theorem holds.

\section{REFERENCES}

[ACG] J. Ashley, E. M. Coven, and W. Geller, Entropy of semipatterns or how to connect the dots to minimizing entropy, Proc. Amer. Math. Soc. 113 (1991), 1115-1121.

[ALM1] Ll. Alsedà, J. Llibre, and M. Misiurewicz, Periodic orbits of maps of $Y$, Trans. Amer. Math. Soc. 313 (1989), 475-538.

[ALM2] __ Combinatorical dynamics and entropy in dimension one, Advanced Series on Nonlinear Dynamics 5, World Scientific, Singapore, 1993.

[AM] Ll. Alsedà and J. M. Moreno, Lower bounds of the topological entropy of maps from $Y, \mathrm{~J}$. Math. Anal. Appl. 155 (1991), 513-530.

[AYj Ll. Alsedà and X. Ye, Minimal sets of maps from Y, J. Math. Anal. Appl. 187 (1994), 324-338.

[Bald1] S. Baldwin, An extension of Sarkovskii's theorem to $n$-od, Ergodic Theory Dynamical Systems 11 (1991), 249-271.

[Bald2] __, On the possible sets of periods for functions on trees, preliminary reports, 1992.

[BC] L. Block and W. A. Coppel, Dynamics in one dimension, Lecture Notes in Math., vol. 1513, Springer-Verlag, 1992.

[BGMY] L. Block, J. Guckenheimer, M. Misiurewicz, and L. S. Young, Periodic points and topological entropy of one dimensional maps, Lecture Notes in Math., vol. 819, Springer, 1980, pp. 18-34. 
[Blok] A. M. Blokh, Periods implying almost all periods, trees with snowflakes, and zero entropy maps, Nonlinearity 5 (1992), 1375-1382.

[FM] J. Franks and M. Misiurewicz, Cycles for disk homeomorphisms and thick trees, Nielsen Theory and Dynamical Systems (C. K. McCord, ed.), Contemp. Math., vol. 152, Amer. Math. Soc., Providence, R.I., 1993, pp. 69-139.

[K] K. Kuratowski, Topology. II, Academic Press, New York and London, 1968.

[DGS] M. Denker, C. Grillenberger, and K. Sigmund, Ergodic theory on compact spaces, Lecture Notes in Math., vol. 527, Springer, Berlin, 1976.

[LM] J. Llibre and M. Misiurewicz, Horseshoes, entropy and periods for graph maps, Topology 32 (1993), 649-664.

[Y] X. Ye, The center and the depth of the center for a tree map, Bull. Austral. Math. Soc. 48 (1993), 347-350.

Department of Mathematics, National University of Singapore, Singapore 0511, RePUBLIC OF SINGAPORE

Section of Mathematics, International Centre for Theoretic Physics, P.O. Box 586, 34100 TRIESTE, ITALY

Current address: Department of Mathematics, University of Science and Technology of China, Hefei, Anhui, 230026, P. R. China 